This is the peer reviewed version of the following article:

Fuertes-Espinosa C., Gómez-Torres A., Morales-Martínez R., Rodríguez-Fortea A., García-Simón C., Gándara F., I maz I., Juanhuix J., Maspoch D., Poblet J.M., Echegoyen L., Ribas X.. Purification of Uranium-based Endohedral Metallofullerenes (EMFs) by Selective Supramolecular Encapsulation and Release. Angewandte Chemie - International Edition, (2018). 57. : 11294 - . 10.1002/anie.201806140,

which has been published in final form at https://dx.doi.org/10.1002/anie.201806140. This article may be used for non-commercial purposes in accordance with Wiley Terms and Conditions for Use of Self-Archived Versions. 


\title{
Purification of Uranium-based Endohedral Metallofullerenes (EMFs) by Selective Supramolecular Encapsulation and Release
}

\author{
Carles Fuertes-Espinosa, ${ }^{[\mathrm{a}]}$ Alejandra Gómez-Torres, ${ }^{[\mathrm{b}]}$ Roser Morales-Martínez, ${ }^{[\mathrm{c}]}$ Antonio Rodríguez- \\ Fortea, ${ }^{[\mathrm{c}]}$ Cristina García-Simón, ${ }^{[\mathrm{a}]}$ Felipe Gándara, ${ }^{[\mathrm{d}]}$ Inhar Imaz, ${ }^{[\mathrm{e}]}$ Judith Juanhuix,${ }^{[\mathrm{f}]}$ Daniel \\ Maspoch, ${ }^{[\mathrm{e}, \mathrm{g}]}$ Josep M. Poblet, ${ }^{[\mathrm{c}]}$ Luis Echegoyen, ${ }^{[\mathrm{b}] *}$ and Xavi Ribas ${ }^{[\mathrm{a}] *}$
}

[*] C. Fuertes-Espinosa, Dr. C. García-Simón, Dr. X. Ribas, QBIS group, IQCC and Dept. Química, Universitat de Girona, Campus de Montilivi, E-17003, Girona, Catalonia, Spain. E-mail: xavi.ribas@udg.edu

A. Gómez-Torres, Prof. L. Echegoyen, Department of Chemistry, University of Texas at El Paso, 500 West University Avenue, El Paso, Texas 79968, United StatesEmail: echegoyen@utep.edu

R. Morales-Martínez, Dr. A. Rodríguez-Fortea, Prof. J.M. Poblet Departament de Química Física i Inorgánica, Universitat Rovira i Virgili, C/Marcel-lí Domingo 1, 43007 Tarragona, Spain.

Dr. F. Gándara, Materials Science Factory, Instituto de Ciencia de Materiales de Madrid (ICMM), Consejo Superior de Investigaciones Científicas (CSIC), Calle Sor Juana Inés de la Cruz, 3, 28049 Madrid, Spain

Dr. I. Imaz, Prof. D.Maspoch Catalan Institute of Nanoscience and Nanotechnology (ICN2), CSIC and BIST, Campus UAB, Bellaterra, 08193 Barcelona, Spain

Dr. J. Juanhuix ALBA Synchrotron, Carrer de la llum 2-26, 08290 Cerdanyola del Vallès, Barcelona, Catalonia, Spain Prof. D. Maspoch ICREA, Pg. Lluís Companys 23, 08010 Barcelona, Spain

\begin{abstract}
Supramolecular nanocapsule $\mathbf{1} \cdot(\mathbf{B A r F}) \mathbf{8}$ is able to sequentially and selectively entrap recently discovered $\mathrm{U}_{2} @ \mathrm{C}_{80}$ and unprecedented $\mathrm{Sc}_{2} \mathrm{CU} @ \mathrm{C}_{80}$, simply by soaking crystals of $\mathbf{1} \cdot(\mathbf{B A r F}) \mathbf{8}$ in a toluene solution of arc-produced soot. These species, selectively and stepwise absorbed by $\mathbf{1} \cdot(\mathbf{B A r F}) \mathbf{8}$, are easily released, obtaining highly pure fractions of $\mathrm{U}_{2} @ \mathrm{C}_{80}$ and $\mathrm{Sc}_{2} \mathrm{CU} @ \mathrm{C}_{80}$ in one step. $\mathrm{Sc}_{2} \mathrm{CU} @ \mathrm{C}_{80}$ represents the first example of a mixed metal actinide-based endohedral metallofullerene (EMF). Remarkably, the host-guest studies revealed that $\mathbf{1} \cdot(\mathbf{B A r F})_{8}$ is able to discriminate EMFs with the same carbon cage but with different encapsulated cluster and computational studies provide support for these observations.
\end{abstract}

The development of novel compounds with unprecedented properties, together with the ability to accommodate unstable metal clusters into their carbon cages, are the main motivations boosting the progress of EMF science. The diversity of entrapped guests imparts versatile electronic and magnetic properties to the EMFs, making them promising materials with potential applications in several fields. ${ }^{[1]}$ Despite the many developments in EMF science, which are mainly focused on lanthanide based compounds, actinide EMFs remain poorly explored. ${ }^{[1 a, 2]}$ In early 1992, Smalley and co-workers detected spectrometrically a series of Uranium-based EMFs, such as $U @ C_{2 n}(2 n=28-72)$ and $U_{2} @ C_{2 n}(2 n=50-$ 
60) for the first time. ${ }^{[3]}$ However, it was not until 2017 that the successful synthesis and complete structural characterization of monometallic actinide EMFs were reported $\left(T h @ \mathrm{C}_{3 \mathrm{v}}(8)-\mathrm{C}_{82}, \mathrm{U} @ \mathrm{D}_{3 \mathrm{~h}}-\mathrm{C}_{74}\right.$, $\mathrm{U} @ \mathrm{C}_{2}(5)-\mathrm{C}_{82}$ and $\left.\mathrm{U} @ \mathrm{C}_{2 \mathrm{v}}(9)-\mathrm{C}_{82}\right),{ }^{[4]}$ and very recently Chen and Echegoyen reported the first dimetallic $\mathrm{U}_{2} @ I_{\mathrm{h}}(7)-\mathrm{C}_{80} \cdot{ }^{[5]}$ In spite of the successful examples in producing monometallic and dimetallic actinide EMFs, ${ }^{[4]}$ it is worth noting that no examples of Mixed-Metal Cluster fullerenes (MMCFs) based on actinides have been reported to date. ${ }^{[1 \mathrm{a}]}$

Generally, the practical application and the study of novel EMFs are hampered by their limited availability. High performance liquid chromatography (HPLC) is by far the most powerful and used technique for the isolation of EMFs. ${ }^{[1 \mathrm{a}, 6]}$ Nevertheless, the low production efficiency of these compounds by arc-discharge methods limits their purification by chromatography, resulting in time-consuming and expensive procedures. Commonly, these drawbacks are even more pronounced for the purification of MMCFs crude soot. ${ }^{[1 \mathrm{a}]}$ As a result of the high complexity of the MMCFs arc-produced soot, composed of several species with the same size and isomeric carbon cages (only differentiated by the internal cluster), the selective separation is a very challenging task. Moreover, their low abundance in soot extracts dramatically hinders the purification of these materials by HPLC, even when running in multi-step or recycling modes.

To circumvent the limitations of HPLC purification, some chemical and electrochemical separation methods have been developed for the isolation of EMFs. ${ }^{[1 \mathrm{a}, 7]}$ To date, the only non-chromatographic strategy attainable for the purification of MMCFs was reported by Stevenson, ${ }^{[8]}$ and it was successfully applied in the separation of $\mathrm{CeLu}_{2} \mathrm{~N} @ \mathrm{C}_{80}$ by combining two chemical methods of purification: successive precipitation of EMFs with a Lewis acid, followed by a stir and filter approach (SAFA). Still, several steps and long reaction times are required. On the other hand, encapsulation of EMFs using self-assembled supramolecular nanocapsules is emerging as a topic of great interest. ${ }^{[9]}$ The design of supramolecular platforms for selective host-guest complexation of EMFs is a potential alternative to HPLC for the purification of these compounds. Recently, our group reported the first example of a supramolecular nanocapsule that allowed the efficient and simple purification of $\mathrm{Sc}_{3} \mathrm{~N} @ \mathrm{C}_{80}$ by selective host-guest complexation. ${ }^{[10]}$

Herein we report the straightforward selective purification of dimetallic and mixed metallic U-based EMFs from a crude soot by host-guest encapsulation in a $\mathrm{Cu}^{\text {II }}$-based tetragonal prismatic nanocapsule 1·(BArF)8. ${ }^{[10]}$ The sequential selective encapsulation of the desired EMFs enables isolation of $U_{2} @ I_{\mathrm{h}}-\mathrm{C}_{80}$ and $\mathrm{Sc}_{2} \mathrm{CU} @ \mathrm{I}_{\mathrm{h}}-\mathrm{C}_{80}$ from arc-produced soot in sequential single steps. Host-guest studies revealed that nanocapsule $\mathbf{1} \cdot(\mathbf{B A r F})_{8}$ is sensitive to the content inside the fullerene cage, being able to distinguish between EMFs with the same carbon cage but different internal clusters. 


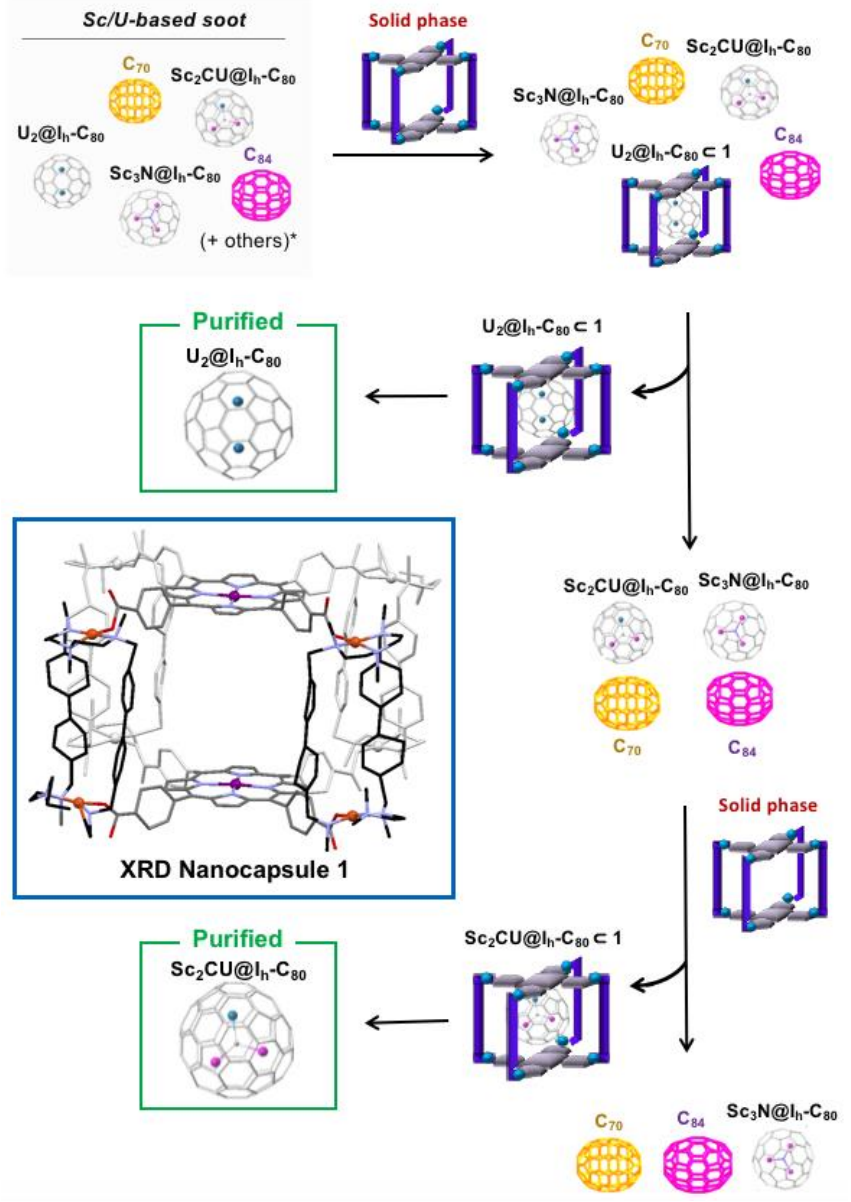

Figure 1. Straightforward purification protocol for selective and sequential encapsulation of $\mathrm{U}_{2} @ \mathrm{C}_{80}$ and

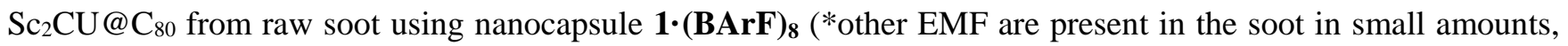
see Fig. 2a). Inset shows a representation of $\mathbf{1}^{8+}$ extracted from SCXRD (CCDC 1845202).

The supramolecular nanocapsule used in the present study was constructed by self-assembly of a dinuclear $\mathrm{Cu}^{\mathrm{II}}$-based macrocyclic synthon and commercially available tetracarboxylate $\mathrm{Zn}{ }^{\mathrm{II}}$-porphyrin, to yield the 3D tetragonal prismatic metal-organic nanocapsule 1•(OTf)8, which after anion exchange yielded $\mathbf{1 \bullet ( B A r F ) 8}$ as a crystalline material. Supramolecular nanocapsule $\mathbf{1} \bullet(\mathbf{B A r F}) \mathbf{8}$ was characterized by high resolution mass spectrometry (HRMS), FT-IR, UV-vis and Single-Crystal X-Ray Diffraction (SCXRD) (Figures S1-S4). As expected, ${ }^{[11]}$ its crystal structure confirmed the formation of a slightly distorted tetragonal prismatic nanocapsule, which is constructed from the two parallel tetracarboxylated $\mathrm{Zn}^{\mathrm{II}}$-porphyrins linked by four dinuclear $\mathrm{Cu}^{\mathrm{II}}$ macrocycles (see Figure 1, Figure $\mathrm{S} 4$ and Table S1).

Soot containing U-based EMFs was produced by vaporization of cored graphite rods containing a mixture of $\mathrm{U}_{3} \mathrm{O}_{8} / \mathrm{Sc}_{2} \mathrm{O}_{3}$ in an arc-discharge reactor under a $\mathrm{He} / \mathrm{H}_{2}$ atmosphere. The resulting soot was extracted 
with $\mathrm{CS}_{2}$ (Soxhlet) during $6 \mathrm{~h}$. After removal of $\mathrm{CS}_{2}$ under vacuum, the dry U/Sc-based soot extract was dissolved in toluene. LDI-TOF (Laser Desorption/Ionization-Time of Flight) analysis of the soot showed a complex mixture containing empty fullerenes (mainly $\mathrm{C}_{70}$ ), U-based EMFs, unknown mixed-metallic compound $\mathrm{Sc}_{2} \mathrm{UC}_{81}$ (spectroscopic and theoretical studies indicate that this compound corresponds to $\mathrm{Sc}_{2} \mathrm{CU} @ \mathrm{I}_{\mathrm{h}}-\mathrm{C}_{80}$, see below) and a family of Sc-based EMFs (Figure 2a). ${ }^{[12]}$

Owing to the enhanced encapsulation selectivity of nanocapsule $1 \bullet(O T f) 8$ towards empty fullerenes and Sc-based fullerenes in solution or solid state ${ }^{[10]}$ crystals of $\mathbf{1} \cdot(\mathbf{B A r F}) \mathbf{8}$ were soaked in a toluene solution of the soot extract. The host-guest complexation reaction was monitored over time by LDI-TOF analysis of the supernatant (Figure 2 and S5). To our surprise, the host-guest reaction resulted in the selective complexation of $\mathrm{U}_{2} @ \mathrm{C}_{80}$, as indicated by an intensity decrease over time of the $\mathrm{U}_{2} @ \mathrm{C}_{80}$ peak only. After 6 h no $\mathrm{U}_{2} @ \mathrm{C}_{80}$ was detected by LDI-TOF, while the rest of the fullerenes and EMFs remained in solution (Figure 2b). Taking advantage of the fact that $\mathrm{U}_{2} @ \mathrm{C}_{80}$ was absorbed in soaked crystals of $\mathbf{1} \cdot(\mathbf{B A r F}) \mathbf{8}$, the host-guest complex was easily isolated by filtration. In addition, a HRMS analysis of the isolated crystals (dissolved in $\mathrm{CH}_{3} \mathrm{CN}$ ) showed the peaks corresponding to $\mathbf{U}_{\mathbf{2}} @ \mathbf{C s 0}_{\mathbf{8 0}} \subset \mathbf{C} \cdot(\mathbf{B A r F}) \mathbf{8}$, confirming the selective binding of $\mathrm{U}_{2} @ \mathrm{C}_{80}$ (Figure S6). Subsequently, the release of the selectively entrapped diuranium EMF was achieved by applying our previously reported solvent-washing protocol (using a 1:1 $\mathrm{CS}_{2}: 0-\mathrm{DCB}$ solvent mixture), ${ }^{[11]}$ exploiting the orthogonal solubility of 1•(BArF)8 and $\mathrm{U}_{2} @ \mathrm{C}_{80}$. The LDI-TOF analysis of the released species showed a single peak at m/z 1436.103, corresponding to $\mathrm{U}_{2} @ \mathrm{C}_{80}$, with an observed isotopic distribution which agrees with the theoretical predictions (Figure 2c). Therefore, highly pure $\mathrm{U}_{2} @ \mathrm{C}_{80}$ was obtained in a single, rapid and operationally simple step, being selectively encapsulated in the presence of many other fullerenes and EMFs. 


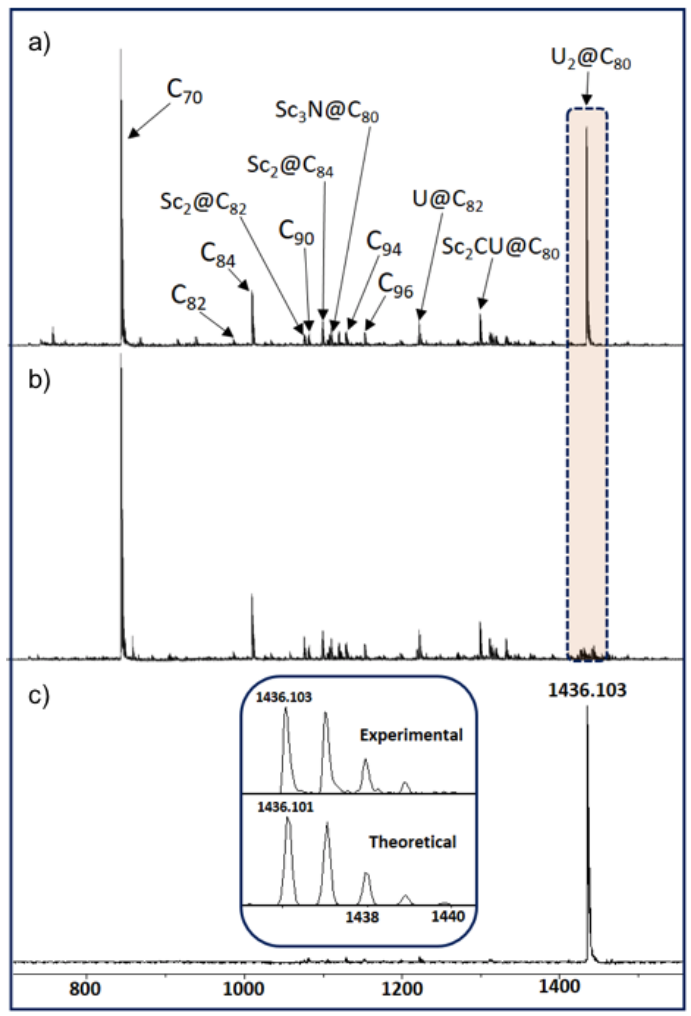

Figure 2. LDI-TOF monitoring of the selective complexation of $\mathrm{U}_{2} @ \mathrm{C}_{80}$ within crystals of $1 \bullet(\mathbf{B A r F})_{8}$ soaked in raw Sc/U soot extract solution in toluene. a) Spectrum of the starting raw $\mathrm{Sc} / \mathrm{U}$ soot extract solution; b) spectrum of the remaining supernatant after soaking crystals of $\mathbf{1} \cdot(\mathbf{B A r F})_{8}$ for $6 \mathrm{~h}$; and $\mathbf{c}$ ) spectrum of the pure $\mathrm{U}_{2} @ \mathrm{C}_{80}$ released from 1•(BArF)8.

After the complete removal of $\mathrm{U}_{2} @ \mathrm{C}_{80}$ from the soot solution mixture, the subsequent encapsulation of $\mathrm{Sc}_{2} \mathrm{CU} @ \mathrm{C}_{80}$ was targeted in order to explore whether a general trend for the selective guest recognition of $\mathrm{C}_{80}$ Uranium-based EMFs existed. An analogous strategy to that employed for the purification of $\mathrm{U}_{2} @ \mathrm{C}_{80}$ was performed, and fresh crystals of $\mathbf{1} \cdot(\mathbf{B A r F}) \mathbf{8}$ were added to the toluene solution containing the remaining mixture of compounds (Figures $2 \mathrm{~b}$ and $3 \mathrm{a}$ ). LDI-TOF analysis revealed a progressive decrease of the peak attributed to $\mathrm{Sc}_{2} \mathrm{CU} @ \mathrm{C}_{80}$ (Figure S7), until its complete disappearance after $1.5 \mathrm{~h}$ (Figure 3b). Confirmation of the selective binding of $\mathrm{Sc}_{2} \mathrm{CU} @ \mathrm{C}_{80}$ within 1•(BArF)8 was obtained by HRMS of the isolated host-guest adduct, showing the corresponding peaks for $\mathbf{S c}_{2} \mathbf{C U} @ \mathbf{C 8 0} \subset \mathbf{1} \cdot(\mathbf{B A r F}) \mathbf{8}$ (Figure S8). The guest selectively absorbed within the crystals of $\mathbf{1}(\mathbf{B A r F}) \mathbf{8}$ was liberated by applying the solvent-washing protocol (using a 1:1 $\mathrm{CS}_{2}: \mathrm{o}-\mathrm{DCB}$ solvent mixture), and LDI-TOF analysis of the guest released from 1•(BArF)8 showed a single peak at m/z=1299.963, corresponding to $\mathrm{Sc}_{2} \mathrm{CU} @ \mathrm{C}_{80}$ (Figure 3c). The separation of this mixed-metallic species is remarkable considering that it could not be 
isolated by HPLC techniques due to its very low concentration and essentially the same retention time to that of $\mathrm{Sc}_{3} \mathrm{~N} @ \mathrm{C}_{80}$ (Figure $\mathrm{S} 9$ ).

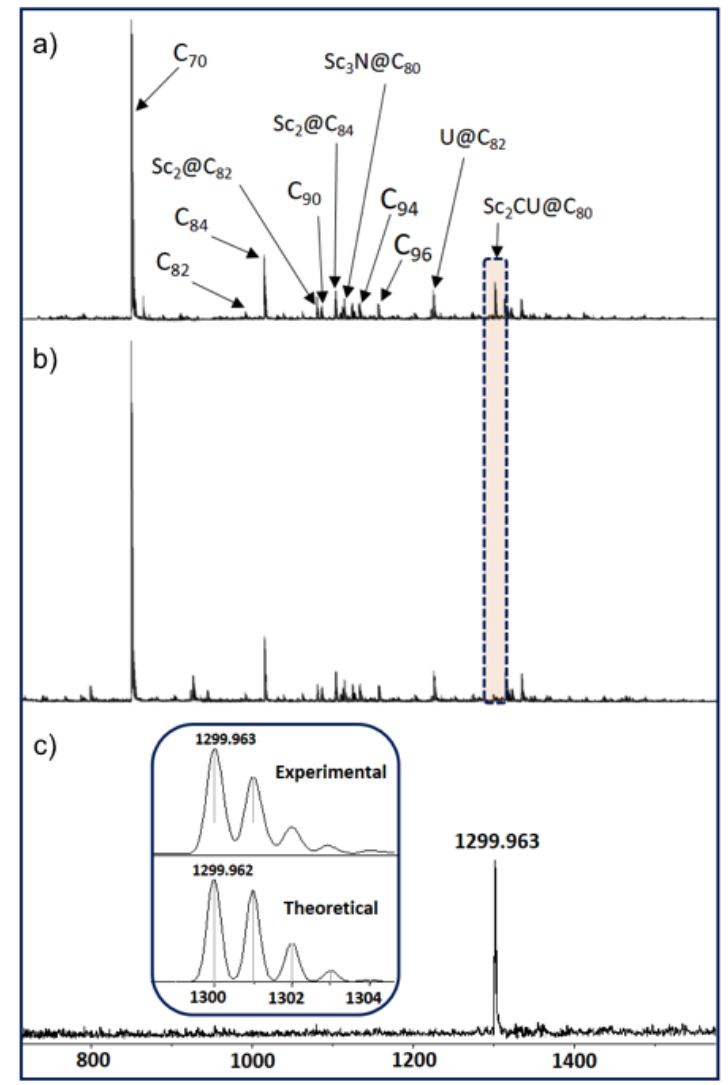

Figure 3. LDI-TOF monitoring of the remaining supernatant during the selective complexation of $\mathrm{Sc}_{2} \mathrm{CU} @ \mathrm{C}_{80}$ within crystals of $1 \bullet(B A r F)_{8}$ soaked in $\mathrm{Sc} / \mathrm{U}$ remaining soot (after the complete removal of $\left.\mathrm{U}_{2} @ \mathrm{C}_{80}\right)$ in toluene. a) Spectrum of the remaining $\mathrm{Sc} / \mathrm{U}$ soot solution; b) spectrum of the remaining supernatant after $1.5 \mathrm{~h}$ of soaking crystals of $\mathbf{1} \cdot(\mathbf{B A r F})_{\mathbf{8}}$; and $\left.\mathbf{c}\right)$ spectrum of the pure $\mathrm{Sc}_{2} \mathrm{CU} @ \mathrm{C}_{80}$ released from 1•(BArF)8.

The UV-vis spectrum of $\mathrm{Sc}_{2} \mathrm{CU} @ \mathrm{C}_{80}$ was rather featureless except for two minor absorption peaks at 600 $\mathrm{nm}$ and $680 \mathrm{~nm}$ (Figure S10). The spectral onset is located at around $900 \mathrm{~nm}$, which indicates a band gap of $1.36 \mathrm{eV}$. Interestingly, these absorption features are very similar to those of $\mathrm{U}_{2} @ I_{\mathrm{h}}-\mathrm{C}_{80},{ }^{[5]}$ further suggesting an identical cage symmetry and charge transfer (in line with DFT calculations, see below), being the internal cluster the only distinctive feature between the two. It thus follows that the selectivity of $1 \bullet(B A r F) 8$ is in response to the nature of the different internal clusters within the carbon cages. Aiming at unraveling the origin of the observed selectivity, crystals of $\mathbf{1} \cdot(\mathbf{B A r F}) \mathbf{8}$ were added to a toluene solution containing an equimolar mixture of $\mathrm{U}_{2} @ I_{\mathrm{h}}-\mathrm{C}_{80}$ and $\mathrm{Sc}_{3} \mathrm{~N} @ I_{\mathrm{h}}-\mathrm{C}_{80}$. These two species were selected because their only difference is the internal cluster. The host-guest complexation was monitored by LDITOF analysis of the supernatant over time, clearly showing the selective uptake of $U_{2} @ I_{\mathrm{h}}-C_{80}$, leaving 
$\mathrm{Sc}_{3} \mathrm{~N} @ \mathrm{I}_{\mathrm{h}}-\mathrm{C}_{80}$ in solution after $5 \mathrm{~h}$ (Figure 4). LDI-TOF analysis of the released species by solventwashing further confirmed the selective binding of $\mathrm{U}_{2} @ I_{\mathrm{h}}-\mathrm{C}_{80}$ (Figure 4 , bottom).

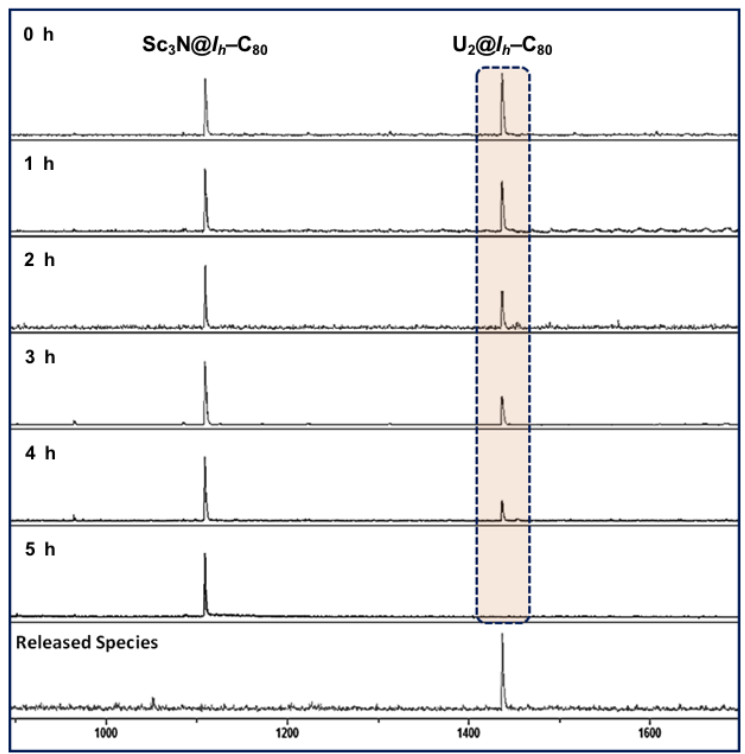

Figure 4. LDI-TOF monitoring of the remaining supernatant during the selective encapsulation of $U_{2} @ I_{\mathrm{h}}-C_{80}$ from an equimolar mixture of $\mathrm{U}_{2} @ I_{\mathrm{h}-}-\mathrm{C}_{80}$ and $\mathrm{Sc}_{3} \mathrm{~N} @ I_{\mathrm{h}}-\mathrm{C}_{80}$ using crystals of 1•(BArF)8. Spectrum at the bottom corresponds to the released pure $\mathrm{U}_{2} @ I_{\mathrm{h}}-\mathrm{C}_{80}$ from $1 \cdot(\mathbf{B A r F})_{8}$.

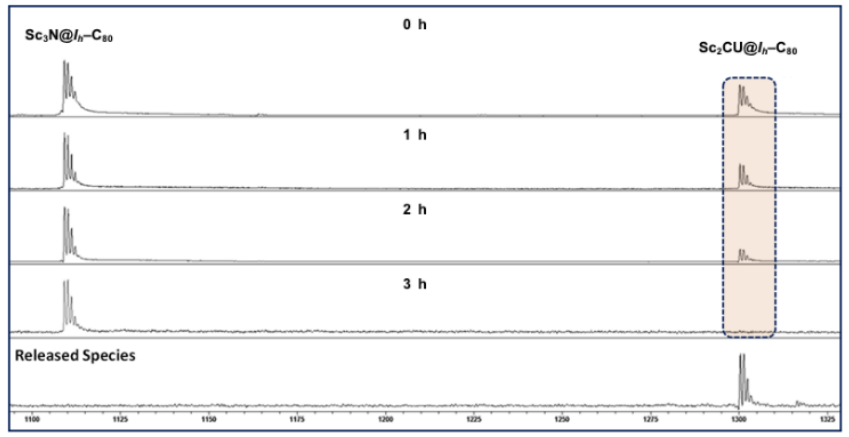

Figure 5. LDI-TOF monitoring of the remaining supernatant during the selective encapsulation of $\mathrm{Sc}_{2} \mathrm{CU} @ I_{\mathrm{h}}-\mathrm{C}_{80}$ from an equimolar mixture of $\mathrm{Sc}_{2} \mathrm{CU} @ \mathrm{I}_{\mathrm{h}}-\mathrm{C}_{80}$ and $\mathrm{Sc}_{3} \mathrm{~N} @ I_{\mathrm{h}-\mathrm{C}_{80}}$ using crystals of $\mathbf{1}(\mathbf{B A r F})_{8}$. Spectrum at the bottom corresponds to the released pure $\mathrm{Sc}_{2} \mathrm{CU} @ \mathrm{I}_{\mathrm{h}}-\mathrm{C}_{80}$ from 1•(BArF).

Finally, the divergent affinity for $\mathrm{Sc}_{2} \mathrm{CU} @ I_{\mathrm{h}}-\mathrm{C}_{80}$ with respect to $\mathrm{Sc}_{3} \mathrm{~N} @ \mathrm{I}_{\mathrm{h}}-\mathrm{C}_{80}$ was also explored by LDI-TOF. Crystals of $\mathbf{1} \cdot(\mathbf{B A r F}) \mathbf{8}$ were soaked in a toluene solution containing one equivalent of $\mathrm{Sc}_{2} \mathrm{CU} @ \mathrm{C}_{80}$ and one equivalent of $\mathrm{Sc}_{3} \mathrm{~N} @ \mathrm{I}_{\mathrm{h}}-\mathrm{C}_{80}$. The host-guest encapsulation was followed over time by LDI-TOF analysis of the supernatant, exhibiting a progressive decrease of the peak attributed to $\mathrm{Sc}_{2} \mathrm{CU} @ \mathrm{C}_{80}$ (Figure 5). After 3h no $\mathrm{Sc}_{2} \mathrm{CU} @ \mathrm{I}_{\mathrm{h}}-\mathrm{C}_{80}$ was detected. Characterization by LDI-TOF of the 
species liberated from 1•(BArF)8 exclusively shows a single peak corresponding to $\mathrm{Sc}_{2} \mathrm{CU} @ \mathrm{I}_{\mathrm{h}}-\mathrm{C}_{80}$, unambiguously indicating the selective formation of complex $\mathbf{S c}_{\mathbf{2}} \mathbf{C U} @ \mathbf{I}_{\mathbf{h}}-\mathbf{C r g}_{\mathbf{8 0}} \subset \mathbf{1} \cdot(\mathbf{B A r F}) \mathbf{8}$ (Figure 5, bottom).

Insight about the structure and electronic properties of unreported $\mathrm{Sc}_{2} \mathrm{UC}_{81}$ compound was gained by computational investigations carried out at the DFT/BLYP/TZP(D3) level. Given that its UV-vis spectrum is virtually identical to those EMFs possessing a $\mathrm{C}_{80}-I_{h}$ cage and by analogy with $\mathrm{Lu}_{2} \mathrm{TiC}_{\mathrm{C}} @ \mathrm{I}_{\mathrm{h}^{-}}$ $\mathrm{C}_{80}$, reported by Popov and co-workers, ${ }^{[2]}$ the internal cluster was assumed to be $\mathrm{Sc}_{2} \mathrm{CU}$ which has a planar structure. As expected, the oxidation state of $U$ is $4+$ and therefore there is a formal electron transfer between host and guest of six electrons. It is worth mentioning that the $\mathrm{U}-\mathrm{C}$ bond length is computed to be $2.070 \AA$, a very short distance essentially identical to the X-ray bond length found very recently for a diuranium carbide cluster $(\mathrm{U}=\mathrm{C}=\mathrm{U})$ stabilized inside $\mathrm{C}_{80}(2.03 \AA) .{ }^{[13]}$ The analysis of the highest occupied orbitals for $\mathrm{Sc}_{2} \mathrm{CU} @ \mathrm{I}_{\mathrm{h}}-\mathrm{C}_{80}$ corroborates that there is a double bond between $\mathrm{U}$ and $\mathrm{C}$ (Figures 6 and $\mathrm{S} 12$ ). The U-C distance is comparable to those found for uranium methylidene complexes $\mathrm{H}_{2} \mathrm{C}=\mathrm{UHX}(\mathrm{X}=\mathrm{F}$, $\mathrm{Cl}$ and $\mathrm{Br})^{[14]}$ and longer than those found for uranium methylidyne molecules $\mathrm{HC} \equiv \mathrm{UX}_{3}(\mathrm{X}=\mathrm{F}, \mathrm{Cl}$ and Br) with $\mathrm{C} \equiv \mathrm{U}$ triple bonds (1.90-1.94 $\AA$, see also Table S2). ${ }^{[15]}$ The average Sc-C distance, $2.024 \AA$, is comparable to that found in the $\square_{3}$-carbido $\mathrm{Sc}_{2} \mathrm{CTi} @ \mathrm{I}_{\mathrm{h}}-\mathrm{C}_{80},{ }^{[16]}$ and somewhat smaller than those in other butterfly-shaped $\mathrm{Sc}_{2} \mathrm{C}_{2}$ clusterfullerenes. ${ }^{[17]}$

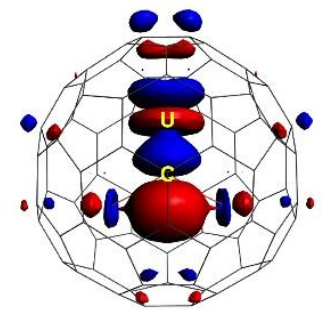

$\sigma$ bond

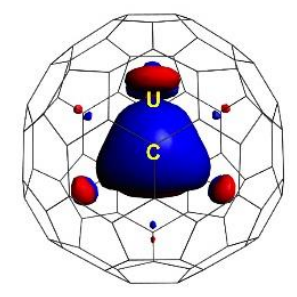

$\pi$ bond

Figure 6. Occupied molecular orbitals for $\mathrm{Sc}_{2} \mathrm{CU} @ \mathrm{I}_{\mathrm{h}}-\mathrm{C}_{80}$ that describe the $\mathrm{U}=\mathrm{C}$ double bond in the $\mathrm{Sc}_{2} \mathrm{CU}$ cluster.

Previous DFT studies of the encapsulation of empty fullerenes by a Pd based nanocapsule showed that there is a strong interaction between the fullerene and the porphyrin. ${ }^{[11]}$ In an effort to understand the basis for the binding selectivity, DFT calculations were performed for simplified model systems in order to evaluate how endohedral fullerenes $\mathrm{U}_{2} @ I_{\mathrm{h}}-\mathrm{C}_{80}$ and $\mathrm{Sc}_{3} \mathrm{~N} @ \mathrm{I}_{\mathrm{h}}-\mathrm{C}_{80}$ interact with the nanocapsule (see SI for computational details). As depicted in Figure 7, the computed $U_{2} @ I_{\mathrm{h}}-C_{80}$ fullerene shows the lowest energy orientation with respect to the two porphyrins of the nanocapsule. The two $\mathrm{U}$ ions are almost perfectly aligned with the $\mathrm{Zn}$ cations of the porphyrins. ${ }^{[5]}$ When the $U$ ions are in a perpendicular orientation the system is destabilized by $1.3 \mathrm{kcal} \cdot \mathrm{mol}^{-1}$ (see Figure $\mathrm{S} 13$ ). Note that whereas in $\mathrm{C}_{60}$ and $\mathrm{C}_{70}$ the carbon atoms are neutral, in $\mathrm{Sc}_{3} \mathrm{~N} @ I_{\mathrm{h}}-\mathrm{C}_{80}$ and $\mathrm{U}_{2} @ I_{\mathrm{h}}-\mathrm{C}_{80}$ there is a formal electron transfer of 6 
electrons between the host and the guest, thus the electron density distribution can play a significant role. ${ }^{[18]}$ The alignment of the two $U$ ions with the porphyrin $\mathrm{Zn}$ cations in $\mathrm{U}_{2} @ I_{\mathrm{h}}-\mathrm{C}_{80}$ seems to be favored by the higher negative charge present on carbon atoms closer to the $\mathrm{U}^{3+}$ ions (see Figures 7 and S14). Therefore, the preferential capture of icosahedral $\mathrm{C}_{80}$ when it possesses two encapsulated $\mathrm{U}$ ions seems to be related to the linear double-conical symmetry of the electron density induced by the presence of the guest ions (Figure 7, left). For $\mathrm{Sc}_{3} \mathrm{~N} @ I_{\mathrm{h}}-\mathrm{C}_{80}$, the electron density is averaged over an equatorial belt and thus it interacts less efficiently with the porphyrin units (Figure 7, right). In line with this, the energy differences among several orientations of the $\mathrm{Sc}_{3} \mathrm{~N}$ cluster and the fullerene with respect to the porphyrins are much smaller, differing only by $0.3 \mathrm{kcal} \cdot \mathrm{mol}^{-1}$ between the perpendicular and parallel cluster orientations (Figure S15).
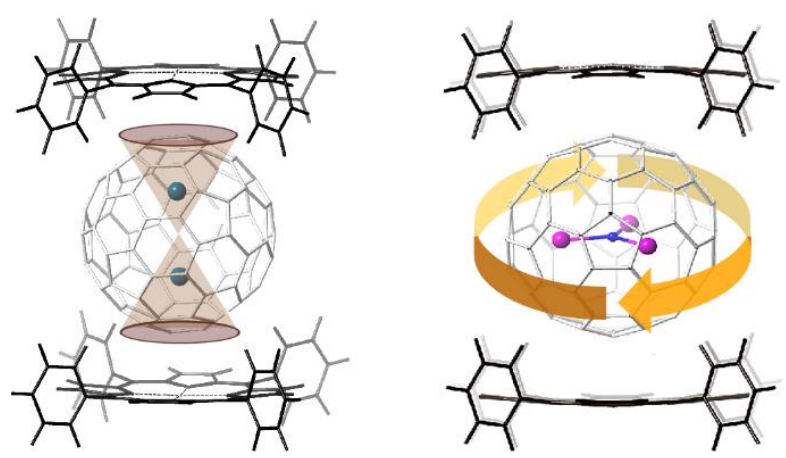

Figure 7. The lowest energy orientation of $\mathrm{U}_{2} @ I_{\mathrm{h}}-\mathrm{C}_{80}$ (left) and $\mathrm{Sc}_{3} \mathrm{~N} @ I_{\mathrm{h}}-\mathrm{C}_{80}$ (right) in a simplified two tetraphenyl-porphyrin (Zn-TPP) model. The electronic density distribution shows a linear double-conical interaction for $\mathrm{U}_{2} @ \mathrm{C}_{80}$, whereas a diffuse, unspecific equatorial belt is found for $\mathrm{Sc}_{3} \mathrm{~N} @ \mathrm{I}_{\mathrm{h}}-\mathrm{C}_{80}$. See Figure $S 14$ for charge distributions and Bader charges on each pentagon.

The computed binding energies (BE) between the carbon cage and the porphyrins for a series of fullerenes are compiled in Table 1 . These values clearly indicate that the porphyrin-fullerene interaction is higher for $\mathrm{Sc}_{3} \mathrm{~N} @ I_{\mathrm{h}}-\mathrm{C}_{80}$ and $\mathrm{U}_{2} @ I_{\mathrm{h}}-\mathrm{C}_{80}$ EMFs. Hence, while the interaction energies for the empty cages range between -43.1 and $-51.5 \mathrm{kcal} \cdot \mathrm{mol}^{-1}$, depending on the cage and orientation of the fullerene (Table 1 and Figures S16), they decrease down to $-57.2 \mathrm{kcal} \cdot \mathrm{mol}^{-1}$ for $\mathrm{Sc}_{3} \mathrm{~N} @ \mathrm{I}_{\mathrm{h}}-\mathrm{C}_{80}$ and down to $-58.9 \mathrm{kcal} \cdot \mathrm{mol}^{-1}$ for $\mathrm{U}_{2} @ I_{\mathrm{h}}-\mathrm{C}_{80}$ (Figure $\mathrm{S} 17$ ). Thermodynamic stabilities extracted from the DFT calculations are aligned with the experimental observations, though it is worth mentioning that kinetic contributions must be relevant in the selection process of one or another EMF, especially those involved in the diffusion of EMFs in the solid phase. 
Table 1. Binding energies between fullerene and two porphyrins.

\begin{tabular}{|l|c|c|c|c|}
\hline & $\mathrm{C}_{60}$ & $\mathrm{C}_{70}$ & $\begin{array}{c}\mathrm{Sc}_{3} \mathrm{~N} @ I_{\mathrm{h}^{-}} \\
\mathrm{C}_{80}\end{array}$ & $\begin{array}{c}\mathrm{U}_{2} @ I_{\mathrm{h}^{-}} \\
\mathrm{C}_{80}\end{array}$ \\
\hline $\mathrm{BE}^{\mathrm{a})}$ & -51.5 & -48.7 & -57.2 & -58.9 \\
\hline $\mathrm{d}(\mathrm{Zn} \cdots \mathrm{Zn})^{\mathrm{b})}$ & 12.6 & 12.9 & 13.8 & 13.9 \\
\hline
\end{tabular}

a) Binding energies computed at the BLYP/TZP(D3) level are given in $\mathrm{kcal} \cdot \mathrm{mol}^{-1} ; \mathrm{Zn} \cdots \mathrm{Zn}$ separations are in $\AA$. For the optimal orientation of the fullerenes see Figures 7 and S17. Computed structures are given in the SI and a data set collection of computational results is available in the ioChem-BD repository ${ }^{[19]}$ and can be accessed via https://doi.org/10.19061/iochem-bd-2-26.

This work describes a straightforward non-chromatographic purification methodology for the selective isolation of $\mathrm{U}_{2} @ \mathrm{I}_{\mathrm{h}}-\mathrm{C}_{80}$ and $\mathrm{Sc}_{2} \mathrm{CU} @ \mathrm{I}_{\mathrm{h}}-\mathrm{C}_{80}$ contained in Sc/U-based arc-produced crude soot. This new strategy overcomes the tedious and time-consuming limitations of HPLC techniques applied for purifying EMFs, and successfully distinguishes EMFs with identical fullerene cages that differ only by the internal cluster. Our approach consists in a sequential and selective uptake of $\mathrm{U}_{2} @ I_{\mathrm{h}}-\mathrm{C}_{80}$ and $\mathrm{Sc}_{2} \mathrm{CU} @ I_{\mathrm{h}}-\mathrm{C}_{80}$, simply by soaking crystals of nanocapsule $1 \bullet(B A r F) 8$ in a toluene solution of crude soot. $\mathrm{Sc}_{2} \mathrm{CU} @ \mathrm{I}_{\mathrm{h}}-\mathrm{C}_{80}$ is a new type of U-EMFs, being the first example of a mixed metallic actinide-based EMF. DFT studies suggest that a highly directional electron density distribution is the basis to explain the different selectivity. 1•(BArF)8 provides a rapid and efficient purification method for EMFs via strong $\pi$ - $\pi$ stacking interactions. No time-consuming work-up is needed for the isolation of the host-guest complex formed and the liberation of highly pure EMFs is further facilitated by the orthogonal solubility between host and guest.

[**] We acknowledge financial support from GenCat (2017 SGR 264 and 2017 SGR 629) and MINECO-Spain (CTQ2016-77989-P and CTQ2017-87269-P). L.E. thanks the US National Science Foundation (NSF) for generous support of this work under the NSF-PREM program (DMR-1205302) and CHE-1408865. The Robert A. Welch Foundation is also gratefully acknowledged for an endowed chair to LE (Grant AH-0033). X.R. and J.M.P. are also grateful to ICREA foundation for ICREAAcadèmia awards. We acknowledge Miquel Costas for helpful discussions. FG acknowledges MINECO for funding through the "Ramón y Cajal" program.

Keywords: U-based EMF • EMF purification • endohedral metallofullerene • supramolecular capsules • mixed-metal actinide EMF. 


\section{References}

[1] a) S. Yang, T. Wei and F. Jin, Chem. Soc. Rev. 2017, 46, 5005-5058; b) M. D. Shultz, J. C. Duchamp, J. D. Wilson, C.-Y. Shu, J. Ge, J. Zhang, H. W. Gibson, H. L. Fillmore, J. I. Hirsch, H. C. Dorn and P. P. Fatouros, J. Am. Chem. Soc. 2010, 132, 4980-4981.

[2] A. L. Svitova, K. B. Ghiassi, C. Schlesier, K. Junghans, Y. Zhang, M. M. Olmstead, A. L. Balch, L. Dunsch and A. A. Popov, Nat. Commun. 2014, 5, 3568.

[3] T. Guo, M. D. Diener, Y. Chai, M. J. Alford, R. E. Haufler, S. M. McClure, T. Ohno, J. H. Weaver, G. E. Scuseria and R. E. Smalley, Science 1992, 257, 1661-1664.

[4] a) Y. Wang, R. Morales-Martínez, X. Zhang, W. Yang, Y. Wang, A. Rodríguez-Fortea, J. M. Poblet, L. Feng, S. Wang and N. Chen, J. Am. Chem. Soc. 2017, 139, 5110-5116; b) W. Cai, R. MoralesMartinez, X. Zhang, D. Najera, E. L. Romero, A. Metta-Magana, A. Rodriguez-Fortea, S. Fortier, N. Chen, J. M. Poblet and L. Echegoyen, Chem. Sci. 2017, 8, 5282-5290.

[5] X. Zhang, Y. Wang, R. Morales-Martínez, J. Zhong, C. de Graaf, A. Rodríguez-Fortea, J. M. Poblet, L. Echegoyen, L. Feng and N. Chen, J. Am. Chem. Soc. 2018, 140, 3907-3915.

[6] M. N. Chaur, F. Melin, A. L. Ortiz and L. Echegoyen, Angew. Chem. Int. Ed. 2009, 48, 7514-7538.

[7] a) E. A. Sarina, B. Q. Mercado, J. U. Franco, C. J. Thompson, M. L. Easterling, M. M. Olmstead and A. L. Balch, Chem. Eur. J. 2015, 21, 17035-17043; b) S. Stevenson, M. A. Mackey, J. E. Pickens, M. A. Stuart, B. S. Confait and J. P. Phillips, Inorg. Chem. 2009, 48, 11685-11690; c) S. Stevenson, K. Harich, H. Yu, R. R. Stephen, D. Heaps, C. Coumbe and J. P. Phillips, J. Am. Chem. Soc. 2006, 128, 8829-8835; d) B. Elliott, L. Yu and L. Echegoyen, J. Am. Chem. Soc. 2005, 127, 10885-10888; e) S. Stevenson, K. D. Arvola, M. Fahim, B. R. Martin, K. B. Ghiassi, M. M. Olmstead and A. L. Balch, Inorg. Chem. 2016, 55, 62-67; f) M. R. Cerón, F. F. Li and L. Echegoyen, Chem. Eur. J. 2013, 19, 7410-7415; g) Z. Ge, J. C. Duchamp, T. Cai, H. W. Gibson and H. C. Dorn, J. Am. Chem. Soc. 2005, 127, 16292-16298.

[8] S. Stevenson, H. R. Thompson, K. D. Arvola, K. B. Ghiassi, M. M. Olmstead and A. L. Balch, Chem. Eur. J. 2015, 21, 10362-10368.

[9] a) L. P. Hernández-Eguía, E. C. Escudero-Adán, J. R. Pinzón, L. Echegoyen and P. Ballester, J. Org. Chem. 2011, 76, 3258-3265; b) M.-Y. Ku, S.-J. Huang, S.-L. Huang, Y.-H. Liu, C.-C. Lai, S.-M. Peng and S.-H. Chiu, Chem. Commun. 2014, 50, 11709-11712.

[10] C. Fuertes-Espinosa, C. García-Simón, E. Castro, M. Costas, L. Echegoyen and X. Ribas, Chem. Eur. J. 2017, 23, 3553-3557.

[11] C. García-Simón, M. Garcia-Borràs, L. Gómez, T. Parella, S. Osuna, J. Juanhuix, I. Imaz, D. Maspoch, M. Costas and X. Ribas, Nat. Commun. 2014, 5, 5557.

[12] The Sc3N@C80 observed in the initial soot is formed presumably due to traces of nitrogen in the arcing chamber.

[13] Unpublished results.

[14] J. T. Lyon and L. Andrews, Inorg. Chem. 2006, 45, 1847-1852.

[15] J. T. Lyon, H.-S. Hu, L. Andrews and J. Li, Proc. Natl. Acad. Sci. USA 2007, 104, 18919-18924.

[16] K. Junghans, K. B. Ghiassi, N. A. Samoylova, Q. Deng, M. Rosenkranz, M. M. Olmstead, A. L. Balch and A. A. Popov, Chem. Eur. J. 2016, 22, 13098-13107.

[17] R. Valencia, A. Rodríguez-Fortea and J. M. Poblet, J. Phys. Chem. A 2008, 112, 4550-4555.

[18] A. Rodriguez-Fortea, A. L. Balch and J. M. Poblet, Chem. Soc. Rev. 2011, 40, 3551-3563.

[19] M. Álvarez-Moreno, C. de Graaf, N. López, F. Maseras, J. M. Poblet and C. Bo, J. Chem. Inf. Model. 2015, 55, 95-103. 